\title{
Los diferentes tipos de láser y sus aplicaciones en podología
}

\author{
Diferents tipes of laser and podiatry applications \\ Saleta Becerra NoAL \\ Diplomada en Podología \\ Licenciada en Comunicación Audiovisual \\ Experto Universitario en Productos de Ortopedia a Medida \\ Master Universitario en Investigación en Podología \\ Clínica Podológica Podosan
}

\section{Correspondencia:}

Saleta Becerra Noal

República del Salvador 28-7c

E-15770 Santiago de Compostela (A Coruña)

Correo electrónico: saletabecerranoal@hotmail.com

Fecha de recepción: 30 de diciembre de 2015

Fecha de aceptación: 14 de febrero de 2016

La autora declara no tener ningún tipo de interés económico o comercial.

\section{RESUMEN}

Los diferentes tipos de láseres, sobre todo el láser de diodo, irrumpen en la terapéutica podológica para proporcionar una alternativa más de tratamiento en muchas patologías que son el día a día de las consultas. El buen manejo y el conocimiento de sus características son requisitos imprescindibles para no tener efectos secundarios indeseados y poder llevar a cabo tratamientos poco dolorosos, minimizando el tiempo total, y muchas veces proporcionando una solución a diversas patologías.

Palabras clave: Láser; diodo; podología.

\begin{abstract}
Diferent tipes of laser, like diode laser, are a good option of podiatry treatment in many patologies that appears in ours clinics. This option needs from the podiatrist a correct management and a deep knowledge of his use for not have secundary efects and could carry out treatments that are not painful, minimizing total time and providing a solution for many patologies.

Key words: Laser; diode; podiatry.
\end{abstract}

Referencia normalizada: Becerra Noal S. Los diferentes tipos de láser y sus aplicaciones en podología. Rev. Int. Cienc. Podol. 2016; 10(2): 62-69.

Sumario: 1. Introducción. 2. Metodología. 3. Discusión. 4. Conclusiones. Bibliografía. 


\section{INTRODUCCIÓN}

La aplicación láser en podología debe basarse en el conocimiento de su manejo y de los principios fundamentales, tanto físicos como biológicos, que dependen de una serie de factores como la energía luminosa emitida, su longitud de onda, duración o tiempo de exposición y potencia.

Podemos clasificar los láseres en dos grandes grupos pero hay que tener en cuenta que a pesar de su diversidad podríamos realizar un mismo tratamiento con más de un tipo de láser, pero la valoración del profesional determinará cuál considera el más idóneo para ese tratamiento en concreto.

- Láser de baja potencia: se usan por su poder antiinflamatorio, analgésico y acción bioestimulante.

- -Láser de alta potencia: Producen efectos físicos visibles y se emplean como si fueran sustitutos de un bisturí o material rotatorio tradicional, con la ventaja de cauterizar a la vez que corta. Ejemplos: Láser As -Ga, Láser As -Ga -Al, Láser $\mathrm{He}-\mathrm{Ne}$.

En la bibliografía existen descripciones de millares de láseres distintos pero para uso clínico tan sólo unos pocos están comercializados y disponibles para ser usados.
Cada tipo de láser tiene una serie de parámetros que no podremos modificar como son su longitud de onda, la forma de distribución de haz y el tiempo de emisión de ese haz. El láser de diodo que usamos en nuestra práctica clínica es el láser Epic de Biolase 940nm (Figura 1) que posee mayor afinidad por los tejidos con pigmentos como la melanina y la hemoglobina. La longitud de onda es la responsable principal de la interacción láser-tejido, y los efectos que se produzcan dependen mucho de la longitud de onda utilizada. La distribución del haz, formado por fotones, transporta la energía teniendo una mayor concentración en el punto central; esta distribución en el reparto transversal de fotones en el eje de avance del haz es lo que se define como TEM. (TEM=Transverse Electromagnetic Mode). Cuando queremos conseguir un efecto de corte nos interesa un TEM 00 , mientras que si se quiere obtener un efecto terapéutico la elección será el TEM en meseta que genera más acúmulo de calor residual ${ }^{1}$.

El tiempo de emisión del haz es la energía láser que se libera durante un determinado tiempo. En nuestro láser existe la posibilidad de aplicarlo en modo pulsado o modo continuo. Hay que tener en cuenta que el mismo láser actuando sobre el mismo tejido puede producir efectos biológicos diferentes en función de los parámetros con los que se utilice. Ten-

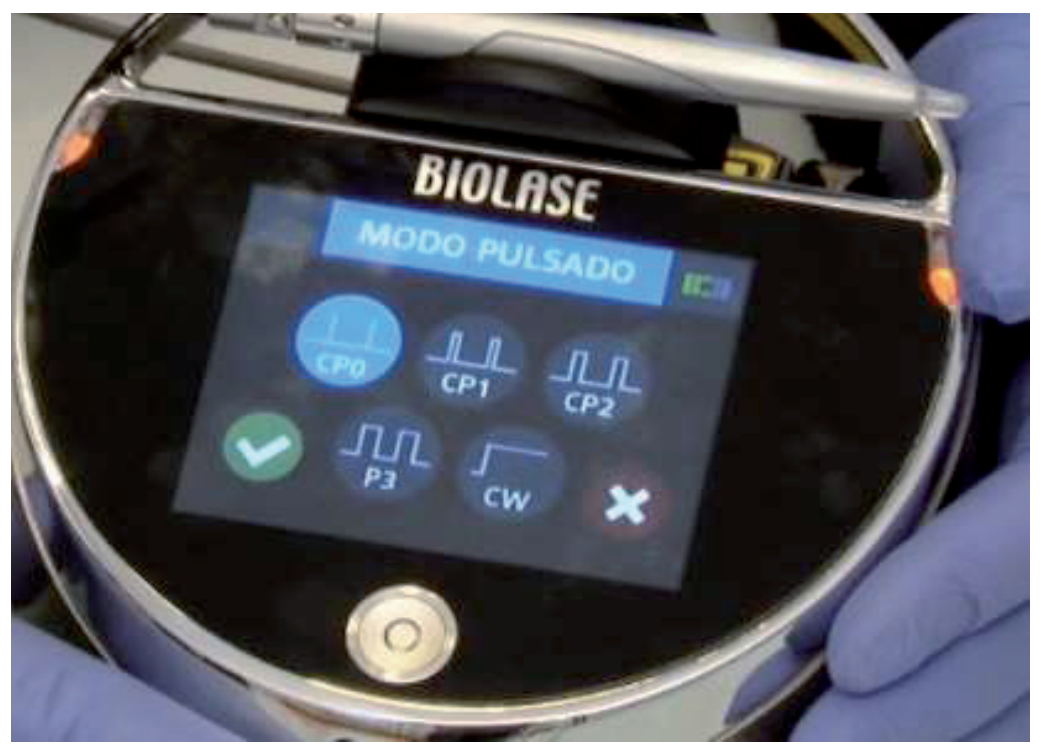

Figura 1. Unidad de Láser de Diodo Biolase. 
dremos que tener en cuenta a la hora de escoger la pieza de mano del láser que la densidad de potencia es inversamente proporcional al radio al cuadrado de la punta.

El láser en función de sus diferentes longitudes de onda tiene diferente poder de penetración (Figura 2) en el tejido humano. Los infrarrojos a pesar de no ser visibles al ojo humano tienen un poder de penetración en los tejidos mayor que alguna luz que es visible ${ }^{2}$.

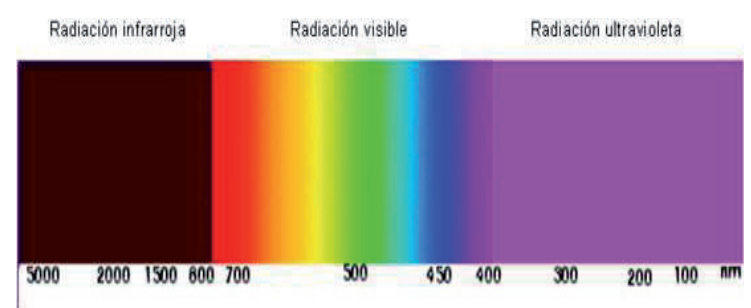

Figura 2. Espectro electromagnético.

\section{METODOLOGÍA}

Se ha realizado la lectura crítica de diversas fuentes bibliográficas en relación a los diferentes tipos de láser y su aplicación en la terapéutica podológica.

Se describe la práctica diaria del uso del láser de diodo en nuestra clínica y su manejo para las patologías mas frecuentes.

\section{Normas de seguridad}

- Los láseres mejor absorbidos producen un rápido incremento de la temperatura en la zona donde se aplica, y este efecto térmico dependerá del tiempo de aplicación.

- No podremos aplicar láser con elementos metálicos por la reflexión del haz.

- Debemos proteger los ojos con las gafas destinadas a tal fin y debidamente homologadas, tanto nosotros como el paciente.

- No aplicar en embarazadas sobre la zona abdominal o lumbar.

- Señalizar debidamente la sala donde se aplica.

- No aplicar cuando existan problemas bacterianos sistémicos, carcinomas ni a alta frecuencia en zonas con mucha melanina por el incremento de la radiación en la zona. En pacientes que estén tomando medicamentos fotosensibles o en zonas fotosensibles. En pacientes epilépticos no sobre el vértex craneal ni en zonas próximas. No aplicar localmente en glándulas endocrinas.

\section{Terapéutica podológica}

El láser en podología tiene diversas aplicaciones pudiendo beneficiarnos de una serie de efectos como la analgesia, antiinflamatorio, antiedematoso o cicatrizante. El efecto fototérmico permite que a baja potencia se obtenga un efecto fotoenergético o bioenergético; mientras que a alta potencia podemos realizar un corte sin casi sangrado pues a medida que corta, coagula la sangre. El efecto fotoquímico hace que la zona donde se aplica libere sustancias como histamina, serotonina y bradicidina; aumenta la producción de ATP intracelular, y estimula la síntesis de $\mathrm{ADN}$, proteínas y enzimas. El efecto fotoeléctrico normaliza el potencial de membrana que actúa sobre la movilidad iónica.

La terapia láser local es un tratamiento que empieza a ganar cada vez más adeptos en las especialidades médicas como una terapia de tratamiento más, siendo la onicomicosis dentro de las patologías dermatológicas una de las patologías más tratadas y con más usuarios. El láser se ha usado en uñas con diversas patologías, consiguiendo una mejora en el aspecto de las uñas de los pies, lo que ha llevado a conseguir que sea un tratamiento aprobado por la FDA. A diferencia de la terapia farmacológica oral, el láser tiene unos efectos secundarios previsibles, pero no existen muchos ensayos aleatorizados y hay pocos estudios aleatorizados que investiguen el efecto del láser en las onicomicosis ${ }^{3}$.

En consulta seguimos el siguiente protocolo de actuación:

- Cultivo de la uña y tratamiento de las posibles causas que han provocado o facilitado el contagio.

- Corte y fresado de la lámina ungueal, dejando la uña lo más corta y sana posible, con el tejido subungueal limpio.

- Limpieza de la zona ungueal y periungueal con desinfectante, que debe ser transparente como alcohol $70 \%$ o agua oxigenada. 
- Con nuestro láser de diodo Epic se usa la punta quirúrgica desechable sin activar a $1 \mathrm{w}$ en modo continuo, lo que permite una buena transmisión de la luz a la lámina y el lecho, haciendo barridos verticales y después horizontales, asegurándonos de aplicar en toda la superficie de la uña (Figura 3). Tras el barrido de todas las uñas con la punta quirúrgica, cambiamos la pieza de mano por el cabezal de bioestimulación, aplicándolo con un protector desechable a $2 \mathrm{w}$ en modo continuo al menos 60 segundos. Existe la posibilidad de usar también la pieza de mano del dolor (más focalizado el haz que emite) a 4w-5w según la sensación del paciente en modo continuo.

Repetimos este mismo protocolo al menos 3 sesiones con un intervalo entre ellas de 7 días, pero en función de la afectación de la uña se necesitan más citas. Después dejamos intervalos de un mes entre aplicaciones si el crecimiento de la raíz está siendo la esperada, hasta la total curación de la uña. La lámina ungueal crece aproximadamente $1 \mathrm{~mm}$ al mes, por lo que realizamos mínimo 1 sesión al mes de lá- ser, combinado con la quiropodia y tratamiento tópico en casa, hasta la total recuperación de la uña. En algunos casos hacemos tratamiento de choque combinado, sobre todo en pacientes que son recurrentes, usando también la terapia antimicótica oral combinada con el tratamiento tópico y láser diodo. En caso de no ver buenos resultados a los tres meses recomendamos al paciente que no continúe con el tratamiento.

Las úlceras de pie diabético tienen diversos métodos convencionales de tratamiento como la limpieza de la herida, el desbridamiento, injertos de piel, tratamientos antibióticos locales y tópicos, tratamientos vasodilatadores sobre todo a nivel de las úlceras vasculares, fármacos para el manejo del dolor y diferentes tipos de vendajes ${ }^{4}$. El láser de baja potencia es uno de los nuevos tratamientos que se incluyen en el manejo terapéutico de las úlceras, proporcionando a través de la energía de la luz, una bioestimulación directa en las células. Esta energía láser es absorbida y estimula las moléculas y los átomos de las células, sin llegar a causar un aumento rápido de la temperatura del tejido donde se aplica ${ }^{5}$.

La terapia láser de baja potencia se usa en clínica como un coadyudante en el tratamiento

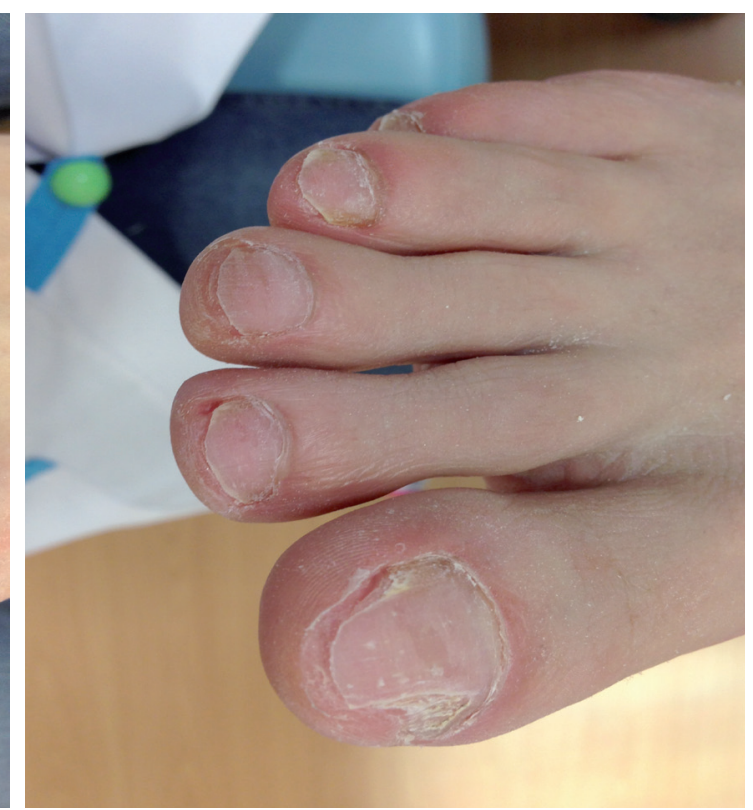

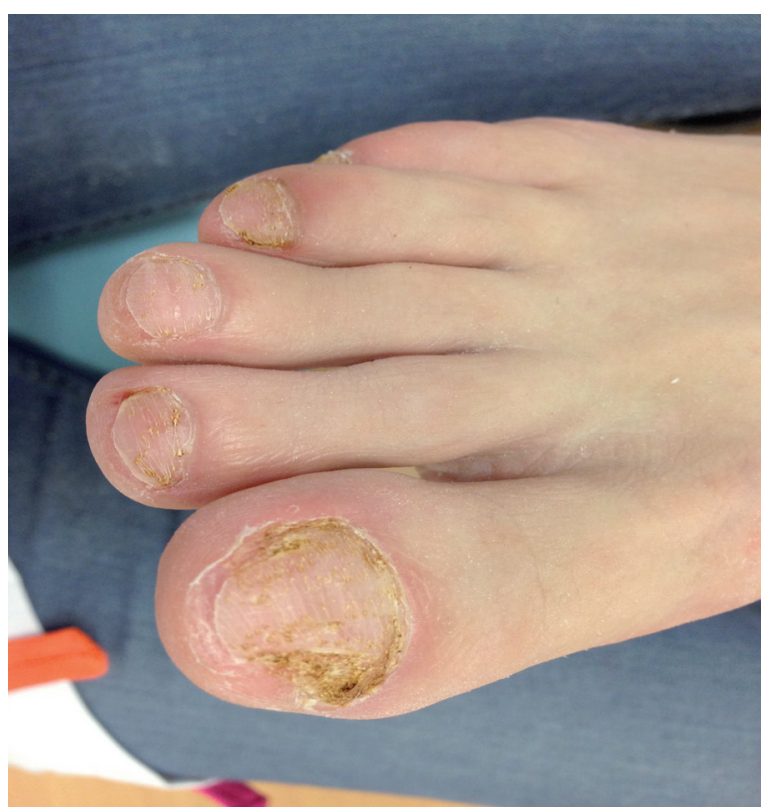

Figura 3. Aspecto de las uñas tras quiropodia antes y después de la aplicación láser. 
del dolor, por su efecto antiinflamatorio, y para acelerar el proceso de curación de diversas patologías musculares ${ }^{6,7}$,quemaduras ${ }^{8}$, heridas postquirúrgicas $^{9,10}$, y úlceras crónicas ${ }^{11}$.

El láser como terapia para la cicatrización de heridas se usa desde 1960 , pero a pesar de llevar tanto tiempo en uso, sus resultados siguen siendo controvertidos. The European Pressure Ulcer Advisory Panel (EPUAP) y The Nacional Pressure Ulcen Advisory Panel (NPUAP) publicaron en 2009 una guía de práctica clínica internacional "Treatment of pressure ulcers: quick reference guide" ${ }^{\prime 13}$.

Diversos estudios evaluaron la eficacia de la terapia láser (810nm) en las úlceras por presión en un estadio III. En este estudio participaron 86 pacientes, que dividieron en dos grupos, donde a uno de ellos se le aplicó además 5 veces a la semana durante 6 semanas terapia láser. Los parámetros que se tuvieron en cuenta para valorar los tratamientos aplicados fueron la reducción del tamaño de la úlcera, si algún paciente desarrolló úlceras en estadio IV. Durante este periodo de tratamiento, el $11 \%$ de los pacientes en el grupo control y el $8 \%$ de los pacientes del grupo tratado también con láser llegaron a un estadio IV en las úlceras por presión. Por lo que consideraron que no había una evidencia científica que justificara el uso de láser de baja potencia como coayudante en el tratamiento en las úlceras por presión ${ }^{14}$.

Otro estudio realizado como por Taradaj en 2013 obtuvo unos resultados donde la terapia láser con una longitud de onda de $658 \mathrm{~nm}$ parecía ser efectiva en el tratamiento de las úlceras por presión, pero la terapia láser con una longitud de onda desde 808 a 940nm no tuvieron el efecto deseado en su estudio ${ }^{15}$.

El protocolo de actuación en las úlceras del pie en nuestra práctica diaria consiste en el tratamiento de la causa y aplicación del láser de diodo diariamente con la pieza de bioestimulación enre 1-3w con barridos durante un ciclo de 360 segundos. Dependiendo de su evolución se pueden dar dos ciclos en una misma sesión e ir espaciando las consultas.

A través del láser de baja potencia se pueden estimular también los puntos de acupuntura. La fibra óptica que emite la luz se pone fija en posición vertical sobre el punto de acupuntura que se quiere estimular. Los efectos terapéu- ticos conseguidos son parecidos a los que se conseguirían con la aguja propia de acupuntu$\mathrm{ra}^{16}$.

A la aplicación del láser en acupuntura se le denomina laserpuntura, donde se aplica radiación láser de forma puntual para conseguir unos efectos locales y sistémicos a partir de efectos primarios físicos, químicos y biológicos que atienden a efectos terapéuticos concretos. También el vocablo se ha usado para nombrar el método de laserterapia sobre puntos de acupuntura, tanto con criterios de la medicina occidental, como de la medicina oriental ${ }^{17}$.

En los últimos años diversos estudios publicados sobre el efecto del láser de baja potencia demuestran que con su aplicación se consigue un aumento de trifosfato de adenosina ${ }^{18}$, una influencia positiva en los fibroblastos ${ }^{19}$ y la síntesis de colágeno ${ }^{20}$ a nivel celular molecular. Existen protocolos clínicos para el uso y manejo del láser de baja potencia para su aplicación en patologías dolorosas y afecciones como la artritis reumatoide, osteoartritis, dolor a nivel cervical, tendinopatías y en dolores crónicos en las articulaciones.

Existen más de 100 estudios publicados, algunos de ellos tipo doble ciego con grupo placebo y grupo control, que demuestran los efectos beneficiosos del uso del láser de baja potencia y su gran efecto antiinflamatorio ${ }^{21}$.

Diversos autores, expresan en sus artículos la rápida mejoría que presentaron sus pacientes con laserterapia y laserpuntura, por sus efectos biológicos a nivel celular y en los tejidos; siendo tratamientos con efecto analgésico, antiinflamatorio, reparador tisular y biomodulador $22,23,24$.

En cirugía el uso del láser está muy estandarizado en diversas especialidades médicas como oftalmología, cirugía vascular u odontología, siendo la podología una de las especialidades que más recientemente lo ha incluido en su práctica diaria. La cirugía ungueal conservadora más exitosa en los últimos años se basa en la avulsión parcial de la uña y la matricectomía química $^{25,26,27,28}$, pues los procedimientos quirúrgicos como la avulsión de la uña, resección lateral, ablación total de la uña y la resección de los tejidos blandos están asociados a un alto índice de recurrencia y morbilidad ${ }^{29}$. Existen dos métodos para realizar una matricectomía, 
que tiene poco índice de recurrencia : mecánica y química, pero en ambas hay una técnica que requiere pericia por parte del profesional, dolor postoperatorio y drenaje prolongado, por lo que muchos autores reportan el uso del láser de $\mathrm{CO} 2$ como una buena alternativa para la matricectomía parcial de la uña ${ }^{30,31,32}$. Este láser de CO2 logra una destrucción más selectiva de la matriz ungueal que la matricectomía química, pero una de sus grandes desvetajas es la dificultad técnica, tiempo de curación prolongado y a nivel estético sin grandes resultados ${ }^{30,32}$.

En cuanto al tratamiento de las verrugas plantares causadas por el virus del papiloma humano, el uso del láser se ha incluido como una opción terapéutica más para ofrecer a nuestros pacientes. Existen diversos tratamientos para destruir la lesión como la crioterapia ${ }^{33}$, curetaje, terapia láser $\mathrm{CO} 2^{34}$, terapia con láser de luz pulsada ${ }^{35}$, electrocirugía y el uso de ácidos tópicos ${ }^{36,37}$, coagulación con infrarojos ${ }^{37}$, aplicación tópica de 5 -fluorouracil ${ }^{39}$, bleomicina intralesional ${ }^{40}$ aunque en España no está dentro de las aplicaciones clínicas de este fármaco, uso de homeopatía tópica y oral...aún así muchas verrugas plantares de resisten a estas terapias.

En nuestra práctica diaria empezamos por tratamientos tópicos con ácido salicílico, cantaridina... y si en un tiempo prudencial no ha remitido la patología ofrecemos el láser de diodo como una terapia más, a veces combinada. Una vez a la semana se desbrida la zona y usamos la pieza quirúrgica con la punta activada (CP2) a 0,8 vatios o lo que el paciente tolere haciendo barridos varias veces cauterizando la zona, después cambiamos la pieza de mano y aplicamos la pieza de bioestimulación entre 1-3 vatios, consiguiendo el efecto fototérmico y fotoquímico.

\section{DISCUSIÓN}

La terapia láser aparece como alternativa a otros tratamientos en la práctica clínica podológica. Es importante conocer su manejo y tomar una serie de precauciones para unos óptimos resultados. Como ventajas podemos destacar su rapidez al obtener resultados y que su aplicación en la mayoría de los pacientes es indolora, además en la onicomicosis es una muy buena alternativa en aquellos pacientes que no pueden tomar los antimicóticos orales, en el tratamiento del dolor para aquellos pacientes que no pueden tomar ningún antiinflamatorio, y en el manejo de las úlceras como coadyudante que acelera de una forma rápida, sin efectos adversos y sin dolor la curación de la misma. En cirugía proporciona un corte rápido, preciso y con la ventaja de la cauterización al mismo tiempo. El mayor inconveniente podría ser su elevado coste al adquirirlo y su mantenimiento, por lo que el precio que debe pagar el paciente por tratamiento también lo es, si lo comparamos con otros tratamientos ofrecidos.

Pensamos que el láser debe acompañarse en todos los casos donde lo apliquemos de un tratamiento de la causa que provoca el problema, para que sus efectos ayuden a la curación de la patología y que no remita.

\section{CONCLUSIONES}

El láser de diodo es de los más utilizados en el campo de la podología, con una longitud de onda, distribución y emisión del haz muy concretos en cada tipo de láser de diodo. $\mathrm{Su}$ buen manejo y conocimiento de las normas de seguridad y su uso en la terapéutica en podología permitirá conseguir pocos o nulos efectos adversos y resultados muy aceptables.

En una clínica de podología el dolor, las onicomicosis, las verrugas plantares o las úlceras son patologías que se diagnostican a diario, entre otras, y el láser de diodo es un buen tratamiento para todas ellas, avalado por múltiples estudios científicos. Se necesitan más investigaciones sobre sus efectos con una muestra de la población mayor para poder crear protocolos de actuación y consenso en su uso en nuestro campo. 


\section{BIBLIOGRAFÍA}

1. Correa, PE. Láser en odontología. CES Odontología. 2002; 15(2): 51-62.

2. Karu T. Mechanisms of low-power laser light action on cellular level. Lasers in Medicine and Dentistry. Rijeka (Croatia): Vitgraf; 2000.

3. Bhatta AK, Huang X, Keyal U, Zhao JJ. Laser treatment for onychomycosis: a review. Mycoses. 2014;57:734-40.

4. Rüttermann M, Maier-Hasselmann A, Grebe BN, Burckhardt M. Klinische Leitlinie Lokaltherapie chronischer Wunden. Bei Patienten mit peripherer arterieller Verschlusskrankheit, chronisch-venöser Insuffizienz und Diabetes mellitus. Deutsches Ärzteblatt. 2013;110(3):25-31.

5. Karu T. Photobiology of low-power laser effects. Health Physics. 1989; 56 (5): 691-704.

6. Leal Junior EC, de Godoi V, Mancalossi JL, Rossi RP, De Marchi T, Parente M, et al. Comparison between cold water immersion therapy (CWIT) and light emitting diode therapy (LEDT) in short-term skeletal muscle recovery after high-intensity exercise in athletes-preliminary results. Lasers Med Sci. 2011; 26: 493-501.

7. Franca CM, de Loura Santana C, Takahashi CB, Alves AN, De Souza Mernick AP, Fernandes KP, et al. Effect of laser therapy on skeletal muscle repair process in diabetic rats. Lasers Med Sci. 2013; 28: 1331-1338.

8. Nunez SC, Franca CM, Silva DFT, Nogueira GE, Prates RA, Ribeiro MS. The influence of red laser irradiation timeline on burn healing in rats. Lasers Med Sci. 2013; 28: 633-641.

9. Pinto FC, Chavantes MC, Pinto NC, Alho EJ, Yoshimura EM, Matushita H, et al. Novel treatment immediately after myelomeningocele repair applying low-level laser therapy in newborns: a pilot study. Pediatr Neurosurg. 2010; 46: 249-254.

10. Dixit S, Maiya A, Rao L, Rao MA, Shastry BA, Ramachandra L. Photobiomodulation by helium neon and diode lasers in an excisional wound model: A single blinded trial. Adv Biomed Res. 2012; 1: 38.

11. Kajagar BM, Godhi AS, Pandit A, Khatri S. Efficacy of low level laser therapy on wound healing in patients with chronic diabetic foot ulcers-a randomised control trial. Indian J Surg. 2012; 74: 359-363.

12. Kaviani A, Djavid GE, Ataie-Fashtami L, Fateh M, Ghodsi M, Salami M, et al. A randomized clinical trial on the effect of low-level laser therapy on chronic diabetic foot wound healing: a preliminary report. Photomed Laser Surg. 2011; 29: 109-114.

13. European Pressure Ulcer Advisory Panel and National Pressure Ulcer Advisory Panel. Treatment of Pressure Ulcers: Quick Reference Guide. Washington, USA: National Pressure Ulcer Advisory Panel; 2009.

14. Lucas C, Van Gemert MJC, De Haan RJ. Efficacy of low-level laser therapy in the management of stage III decubitus ulcers: a prospective, observer-blinded multicentre randomised clinical trial. Lasers in Medical Science. 2003;18(2):72-77.

15. Taradaj, J, Halski, T, Kucharzewski, M, Urbanek, T, Halska, U y Kucio, C. Effect of laser irradiation at different wavelengths $(940,808$, and $658 \mathrm{~nm})$ on pressure ulcer healing: results from a clinical study. Evidence-Based Complementary and Alternative Medicine, 2013.

16. Ebrahimi T, Moslemi N, Rokn AR, Heidari M, Nokhbatolfoghahaie H, Fekrazad R. The influence of low-intensity laser therapy on bone healing. Journal of Dentistry. 2012;9(4):238-248.

17. Delgado Del Busto CA. La laserpuntura una modalidad terapéutica de la Medicina Tradicional Asiática. CENAMENT. [Internet] 2005 [Acceso 1 OCTURE 2015]. Disponible en: http://www.sld.cu/galerias/pdf/sitios/mednat/laserpuntura_carlos.pdf.

18. Hashmi JT, Huang YY, Osmani BZ, Sharma SK, Naeser MA, Hamblin MR. Role of low-level laser therapy in neurorehabilitation. PM R. 2010;2(12 Suppl 2): 292-305.

19. van Breugel HH, Bär PR. Power density and exposure time of He-Ne laser irradiation are more important than total energy dose in photo-biomodulation of human fibroblasts in vitro. Lasers Surg Med. 1992;12(5):528-537.

20. Lam TS, Abergel RP, Meeker CA, Castel JC, Dwyer RM, Uitto J. Laser stimulation of collagen synthesis in human skin fibroblast cultures. Lasers Life Sci. 1986;1:61-77.

21. Bjordal JM, Johnson MI, Iversen V, Aimbire F, Lopes-Martins RA. Low-level laser therapy in acute pain: a systematic review of possible mechanisms of action and clinical effects in randomized placebocontrolled trials. Photomed Laser Surg. 2006;24(2):158-168.

22. Karu T. Low-power laser effects. Lasers in Medicine. Boca Raton: CRC Press; 2002.169.

23. Orellana Molina A, Hernández Díaz A, González Méndez B. Experiencias en la aplicación de la tera- 
pia láser en la Clínica Central "Cira García". Rev Ciencias [revista en Internet] 2007. [acceso 24 Octubre de 2015]. Disponible en: http://www.ilustrados.com/publicaciones/EEAuApAZVkyXfvcKuX.php.

24. González Méndez B, Hernández Díaz A, Estévez Perera A. Tratamiento del herpes simple labial con láser de baja potencia. Rev Colombia Médica. 2008;39:175-81.

25. Altinyazar HC. Surgical treatment of ingrown nails. Turkiye Klinikleri J Int Med Sci 2005;1:60-2.

26. Ozdemir E, Bostaci S, Ekmekci P, Gurgey E. Chemical matricectomy with 10\% sodium hidroxide for the treatment of ingrowing toenails. Dermatol Surg. 2004; 30:26-31.

27. Kim SH, Ko HC, Oh CK, Kwon KS, Kim MB. Trichloroacetic acid matricectomy in the treatment of ingrowing toenails. Dermatol Surg. 2009;35:973-9.

28. Kocyigit P, Bostanci S, Ozdemir E, Gürgey E. Sodium hydroxide chemical matricectomy for the treatment of ingrown toenails: Comparison of three different application periods. Dermatol Surg. $2005 ; 31: 744-7$.

29. Yang KC, Li YT. Treatment of recurrent ingrown great toenail associated with granulation tissue by partial nail avulsion followed by matricectomy with sharpulse carbon dioxide laser. Dermatol Surg. 2002;28:419-21.

30. Serour, F. Recurrent ingrown big toenails are efficiently treated by $\mathrm{CO} 2$ laser. Dermatologic surgery. 2002; 28(6): 509-512.

31. Lin YC, Su HY. A surgical approach to ingrown nail: partial matricectomy using CO2 laser. Dermatol Surg. 2002;28:578-80.

32. Ozawa T, Nose K, Harada T, Muraoka M, Ishii M. Partial matricectomy with a CO2 laser for ingrown toenail after nail matrix staining. Dermatol Surg. 2005; 31:302-5.

33. Bourke JF, Berth-Jones J, Hutchinson PE. Cryotherapy of common viral warts at intervals of 1, 2, and 3 weeks. Br J Dermatol. 1995;132:433-436.

34. Tan OT, Hurwitz TM, Stafford TJ. Pulsed dye laser treatment of recalcitrant verrucae, a preliminary report. Lasers Surg Med. 1993;13:127-137.

35. Logan RA, Zachary CB. Outcome of carbon dioxide laser therapy from persistent cutaneous viral warts. Br J Dermatol. 1989;21:99.

36. Hirose, A y Kobayashi, KF. Formation of hybrid clad layers by laser processing. ISIJ international. 1995; 35(6): 757-763.

37. Bunney MH, Nolan MW, Williams DA. An assessment of methods of treating viral warts by comparative treatment trials based on standard design. Br J Dermatol. 1976;94:667-680.

38. Halasz CL. Treatment of common warts using the infrared coagulator. J Dermatol Surg Oncol. 1994;20:252-256.

39. Brodell RT, Breadle DL. The treatment of palmar and plantar warts using natural alpha interferon and a needless injector. Dermatol Surg. 1995;21:213-218.

40. James MP, Collier PM, Aherne W, et al. Histologic, pharmacologic, and immunocytochemical effects of injection of bleomycin. J Am Acad Dermatol. 1993;28:933-937. 